\title{
Mode growth and competition in the x-ray free-electron laser oscillator start-up from noise
}

\author{
R. R. Lindberg* and K.-J. Kim \\ Advanced Photon Source, Argonne National Laboratory, Argonne, Illinois 60439, USA
}

(Received 11 March 2009; published 2 July 2009)

\begin{abstract}
We describe the radiation properties of an x-ray free-electron laser (FEL) oscillator, beginning with its start-up from noise through saturation. We first decompose the initially chaotic undulator radiation into the growing longitudinal modes of the composite system consisting of the electron beam, the undulator, and the Bragg mirror resonator cavity. Because the radiation initially comprises several modes whose growth rates are comparable, we find that only after many oscillator passes is the output pulse dominantly characterized by the lowest-order Gaussian mode. We verify our analytic results with a novel, reduced one-dimensional FEL code (derived in the text), and with two-dimensional FEL simulations. Understanding the full longitudinal structure during the initial amplification will be critical in assessing the tolerances on the electron beam, undulator, and optical cavity required for robust operation.
\end{abstract}

DOI: 10.1103/PhysRevSTAB.12.070702

PACS numbers: 41.60.Cr, 52.59.Rz

\section{INTRODUCTION}

The basic principles of the free-electron laser (FEL) oscillator are well known (see, e.g., [1]), involving the successive FEL gain of radiation confined in an optical cavity. Over the past few decades several FEL oscillators have been designed and built as sources of intense infrared radiation. Extending the FEL oscillator concept to the $\mathrm{x}$-ray part of the spectrum was first suggested by Colella and Luccio [2] over 25 years ago. Their design proposed using curved silicon mirrors that Bragg reflect $\mathrm{x}$ rays over a narrow bandwidth near $1 \mathrm{keV}$ and called for a highbrightness electron beam; however, the understanding of the electron beam requirements appropriate for gain at $\mathrm{x}$-ray energies was not sufficiently well understood at the time, and the proposal remained largely speculative.

Recently, a concrete set of realizable parameters for a FEL oscillator has been proposed [3] that has the capability of producing hard $\mathrm{x}$ rays with energies between 5 and $20 \mathrm{keV}$. The FEL design uses an ultralow-emittance ( $\sim 0.1 \mathrm{~mm}$ mrad $)$, low-charge $(\sim 40 \mathrm{pC})$ electron beam and a resonator cavity formed using high-reflectivity, narrow-bandwidth Bragg mirrors made of perfect sapphire or diamond crystals [4] (for general results on X-ray optics see, e.g., [5] and references cited therein). It was shown in Ref. [3] that the per-pass FEL gain $g \sim 0.3-0.5$ can readily overcome the cavity losses $\alpha \sim 0.1-0.25$. Such a device is predicted to provide transform-limited, picosecond $\mathrm{x}$-ray pulses with megawatts of power at repetition rates $\sim 1 \mathrm{MHz}$, thereby serving as a complementary source to those based on self-amplified spontaneous emission, such as Linac Coherent Light Source (LCLS) [6].

*lindberg@aps.anl.gov
We consider in this paper three different photon energies: 5-, 12-, and 20-keV, each of which has a central radiation frequency $\omega_{0}$ and mirror bandwidth $\sigma_{\omega}$ that match the theoretical values for the appropriate Bragg reflection in a perfect diamond crystal. One of the two mirrors is chosen to be thin enough to couple out $4 \%$ of the cavity radiation, while total cavity losses $\alpha=0.15$ is assumed for each scenario. (Note that the absorptive losses for two perfect diamond crystals is calculated to be $\leqslant 2 \%$, leaving an additional $\sim 10 \%$ loss for the grazing incidence focusing mirrors.) Furthermore, for this study we have fixed the peak beam current $I_{\text {peak }}$, mean energy $\gamma_{0} m_{e} c^{2}$ (with $m_{e}, \gamma_{0}, c$ being the electron mass, mean Lorentz factor, and the speed of light, respectively), and relative energy spread $\Delta \gamma / \gamma_{0}$ to be $10 \mathrm{~A}, 7 \mathrm{GeV}$, and $0.02 \%$, respectively. The rest of the parameters have been chosen such that the per-pass FEL gain $g \sim 0.3-0.45$, and are listed in Table I. For the electron beam, the transverse

TABLE I. Possible undulator and beam parameters for three different radiation wavelengths produced by a 7-GeV beam with $I_{\text {peak }}=10 \mathrm{~A}$ and $\Delta \gamma / \gamma_{0}=0.02 \%$. The saturated power $P_{\text {sat }}$ given by our 1D code and the 2D code GINGER were determined assuming mirror losses $\alpha=15 \%$.

\begin{tabular}{lccc}
\hline \hline Parameter & $5 \mathrm{keV}$ & $12 \mathrm{keV}$ & $20 \mathrm{keV}$ \\
\hline$\lambda_{u}(\mathrm{~cm})$ & 2.24 & 1.76 & 1.50 \\
$N_{u}$ & 1000 & 3000 & 3000 \\
$K$ & 2.50 & 1.51 & 1.05 \\
$z_{\beta}(\mathrm{m})$ & 5.0 & 9.0 & 10.0 \\
$\epsilon_{x}(\mathrm{~mm} \mathrm{mrad})$ & 0.2 & 0.2 & 0.1 \\
$g$ & 0.42 & 0.34 & 0.28 \\
$\sigma_{\omega} / \omega_{0}$ & $7.9 \times 10^{-6}$ & $8.4 \times 10^{-7}$ & $1.2 \times 10^{-7}$ \\
$P_{\text {sat }}(1 \mathrm{D})$ & $159 \mathrm{MW}$ & $30 \mathrm{MW}$ & $6.2 \mathrm{MW}$ \\
$P_{\text {sat }}(2 \mathrm{D})$ & $121 \mathrm{MW}$ & $28 \mathrm{MW}$ & $10 \mathrm{MW}$ \\
\hline \hline
\end{tabular}


emittance $\epsilon_{x}=0.1-0.2 \mathrm{~mm} \mathrm{mrad}$, while the focusing parameter, defined via the beam size at focus $\sigma_{x}$ via $z_{\beta} \equiv$ $\sigma_{x}^{2} / \epsilon_{x}$, is chosen to maximize $g$. Note that the number of undulator periods $N_{u} \sim 10^{3}$, the undulator wavelength $\lambda_{u} \sim 2 \mathrm{~cm}$, while the undulator parameter $K \equiv e B / k_{u} m_{e} c$ ( $B$ is the peak undulator magnetic field, $k_{u} \equiv 2 \pi / \lambda_{u}$, and $e$ is the unit electric charge) is chosen to satisfy the FEL resonance condition $\lambda \equiv 2 \pi c / \omega_{0}=\lambda_{u}\left(1+K^{2} / 2\right) / 2 \gamma_{0}^{2}$ at an undulator gap of $5 \mathrm{~mm}$ for a neodymium-iron-boron permanent magnet [7].

Table I also lists the intercavity saturated power $P_{\text {sat }}$ as predicted by our reduced, one-dimensional (1D) model (described more fully in Sec. II), and that given by the two-dimensional (2D) code GINGER [8]. Note that $P_{\text {sat }}$ (1D) is within $30 \%$ of the $2 \mathrm{D}$ predictions after $\sim 8$ decades of exponential growth. By coupling $4 \%$ of this power out of the cavity via the thin mirror, the FEL source is predicted to provide $0.4,1.1$, and $4.8 \mathrm{MW}$ of peak power at 20,12 , and $5 \mathrm{keV}$, respectively, corresponding to $\sim 10^{8}-10^{9}$ photons per pulse.

This paper elaborates on the physics of the x-ray FEL oscillator from its initial start-up from particle shot noise through the nonlinear saturation in terms of the supermodes $[9,10]$ (i.e., the growing eigenmodes) of the system. Throughout the paper we compare our analytic results with those obtained using a reduced, one-dimensional FEL code that we derive in Sec. II. The model equations for this code include the lowest-order effects of energy spread, transverse beam emittance, and radiation diffraction, and has demonstrated reasonable agreement with the results of both the two-dimensional code GINGER [8] and the threedimensional code GENESIS [11]. Having described our numerical code, we then proceed in Sec. III to derive and analytically solve the supermode equation governing the radiation profile before nonlinear saturation of gain. These solutions specify how the effective single-pass FEL gain depends upon the duration of the electron beam, the spectral bandwidth of the Bragg mirrors, and the pass-to-pass timing of the electron bunches. We then show how these modes are initially seeded by the chaotic undulator radiation, resulting in the competition between various longitudinal modes. We conclude with some results obtained for possible 5-, 12-, and 20-keV radiation sources based on an FEL oscillator driven by a 7-GeV high-brightness electron beam.

\section{A REDUCED, ONE-DIMENSIONAL SET OF FEL EQUATIONS}

In this section we reduce the three-dimensional FEL equations to a 1D set by integrating the transverse electron orbits over their unperturbed trajectories and by assuming that the transverse radiation profile is a Gaussian mode whose Rayleigh range $z_{R}$ is dictated by the cavity geometry. To begin, we consider the single-particle equations of motion for the $j$ th electron assuming that there is no external electron beam focusing and ignoring the natural undulator focusing (see, e.g., [12] and references cited therein):

$$
\begin{gathered}
\frac{d}{d z} \theta_{j}=2 k_{u} \eta_{j}-\frac{k_{0}^{2}}{2} \boldsymbol{p}_{j}^{2} \\
\frac{d}{d z} \eta_{j}=\frac{e K[J J]}{2 \gamma_{0}^{2} m_{e} c^{2}}\left(E e^{i \theta_{j}}+E^{*} e^{-i \theta_{j}}\right) \\
\frac{d}{d z} \boldsymbol{x}_{j}=\boldsymbol{p}_{j} \\
\frac{d}{d z} \boldsymbol{p}_{j}=\mathbf{0} .
\end{gathered}
$$

In Eqs. (1)-(4) the electron coordinates include the ponderomotive phase in the wave $\theta_{j} \equiv\left(k_{0}+k_{u}\right) z-c k_{0} t$ (with $k_{0} \equiv \omega_{0} / c$ being the resonant radiation wave vector), the dimensionless energy spread $\eta_{j} \equiv\left(\gamma_{j}-\gamma_{0}\right) / \gamma_{0}$, the transverse position $\boldsymbol{x}_{j}$, and the dimensionless transverse momentum $\boldsymbol{p}_{j}$. The electrons couple to the complex electric field $E$ via Eq. (2), where $[J J]$ is the standard Bessel function factor

$$
[J J] \equiv J_{0}\left(\frac{K^{2}}{4+2 K^{2}}\right)-J_{1}\left(\frac{K^{2}}{4+2 K^{2}}\right) .
$$

We will find it convenient to introduce the single-particle electron distribution function on phase space $f(\theta, \boldsymbol{x}, \eta, \boldsymbol{p}, z)$, normalized such that integrating $f$ over all space is unity. From the conservation equation $d f / d z=$ 0 and the equations of motion (1)-(4), we find that the distribution function obeys the following collisionless Boltzmann equation:

$$
\begin{aligned}
{\left[\frac{\partial}{\partial z}+\left(2 k_{u} \eta-\right.\right.} & \left.\frac{1}{2} k_{0}^{2} \boldsymbol{p}^{2}\right) \frac{\partial}{\partial \theta}+\boldsymbol{p} \cdot \frac{\partial}{\partial \boldsymbol{x}} \\
& \left.+\frac{e K[J J]}{2 \gamma_{0}^{2} m_{e} c^{2}}\left(E e^{i \theta}+\text { c.c. }\right) \frac{\partial}{\partial \eta}\right] f=0 .
\end{aligned}
$$

Meanwhile, the radiation amplitude $E$ obeys the paraxial wave equation driven by the averaged FEL current

$$
\begin{aligned}
& {\left[\frac{\partial}{\partial z}+k_{u} \frac{\partial}{\partial \theta}+\frac{1}{2 i k_{0}} \nabla_{\perp}^{2}\right] E(\theta, \boldsymbol{x}, z)} \\
& =-\frac{e K[J J]}{4 \epsilon_{0} \gamma_{0}} \frac{N_{e}}{2 \pi} \int d \theta d \eta d \boldsymbol{p} e^{-i \theta} f(\theta, \boldsymbol{x}, \eta, \boldsymbol{p}, z),
\end{aligned}
$$

where $N_{e}$ is the total number of electrons in the bunch and $\epsilon_{0}$ is the permittivity of free space. The FEL set of equations (5) and (6) is our starting point for deriving a reduced, quasi-one-dimensional description of the small-gain FEL oscillator. To obtain the reduction, we assume that the solution of Eq. (5) may be factorized into longitudinal and transverse components as 


$$
f(\theta, \boldsymbol{x}, \eta, \boldsymbol{p}, z)=\frac{\tilde{f}(\theta, \eta, z)}{4 \pi^{2} \sigma_{x}^{2} \sigma_{p}^{2}} \exp \left[-\frac{(\boldsymbol{x}-z \boldsymbol{p})^{2}}{2 \sigma_{x}^{2}}-\frac{\boldsymbol{p}^{2}}{2 \sigma_{p}^{2}}\right]
$$

and eliminate the transverse degrees of freedom by integrating the resulting Boltzmann equation over $\boldsymbol{x}$ and $\boldsymbol{p}$. While the momentum integral is trivial, the spatial integral evaluates the overlap of the transverse electric field profile with that of the distribution function. To obtain a closedform expression, we expand $E$ using the vacuum (i.e., source-free) transverse modes of the paraxial equation, appropriate for small single-pass gain. For simplicity we suppress any azimuthal dependence, expanding $E$ in terms of the Gauss-Laguerre functions as

$$
\begin{aligned}
E(\theta, \boldsymbol{x}, z)= & \sum_{q} \frac{E_{q}(\theta, z)}{\sqrt{1+z^{2} / z_{R}^{2}}} L_{q}\left(2 \boldsymbol{x}^{2} / w^{2}\right) \\
& \times \exp \left[-\frac{\boldsymbol{x}^{2}}{w^{2}}\left(1-\frac{i z}{z_{R}}\right)-i \psi_{q}\right],
\end{aligned}
$$

where $E_{q}(\theta, z)$ are the transverse mode coefficients, $L_{q}(x)$ is the Laguerre polynomial of order $q$, the radiation waist $w$ is defined via $w^{2} \equiv w_{0}^{2}\left(1+z^{2} / z_{R}^{2}\right)$ for the focal spot $w_{0}$ and the Rayleigh range $z_{R} \equiv k_{0} w_{0}^{2} / 2$, and $\psi_{q} \equiv$ $(2 q+1) \arctan \left(z / z_{R}\right)$ is the Gouy phase. We complete the dimensional reduction by assuming that the ponderomotive force acting on the electrons is dominated by the lowest-order Gaussian mode whose mode coefficient is $E_{0}$. Thus, we approximate the electric field amplitude $E$ in the Boltzmann equation (5) by

$$
E(\theta, \boldsymbol{x}, z)=\frac{E_{0}(\theta, z)}{1+i z / z_{R}} \exp \left[-\frac{\boldsymbol{x}^{2}}{w_{0}^{2}\left(1+i z / z_{R}\right)}\right],
$$

where we have used

$$
e^{-i \psi_{0}}=e^{-i \arctan \left(z / z_{R}\right)}=\frac{1-i z / z_{R}}{\sqrt{1+z^{2} / z_{R}^{2}}}
$$

to simplify the expression. Using the approximate radiation field (9) and the factorized distribution (7), we integrate over the transverse phase-space dimensions $\boldsymbol{x}$ and $\boldsymbol{p}$ in the collisionless Boltzmann equation (5) to obtain

$$
\begin{aligned}
\left\{\frac{\partial}{\partial z}+\right. & \left(2 k_{u} \eta-\frac{1}{2} k_{0}^{2} \sigma_{p}^{2}\right) \frac{\partial}{\partial \theta} \\
& \left.+\frac{e K[J J]}{2 \gamma_{0}^{2} m_{e} c^{2}}\left[\frac{w_{0}^{2}}{\mu(z)} E_{0}(z, \theta) e^{i \theta}+\text { c.c. }\right] \frac{\partial}{\partial \eta}\right\} \tilde{f}=0,
\end{aligned}
$$

where we have defined the complex reduction parameter

$$
\mu(z) \equiv 2 \sigma_{x}^{2}\left(1+z^{2} / z_{\beta}^{2}\right)+w_{0}^{2}\left(1+i z / z_{R}\right),
$$

and recall that the electron beam focusing parameter is related to the beam emittance $\varepsilon_{x}$ and minimal waist $\sigma_{x}$ by $z_{\beta} \equiv \sigma_{x}^{2} / \varepsilon_{x}$. We can now read off the reduced particle equations of motion from the integrated collisionless Boltzmann equation (10); the equations for the $j$ th electron are

$$
\begin{gathered}
\frac{d}{d z} \theta_{j}=2 k_{u} \eta_{j}-\frac{1}{2} k_{0} \sigma_{p}^{2} \\
\frac{d}{d z} \eta_{j}=\frac{e K[J J]}{2 \gamma_{0}^{2} m_{e} c^{2}}\left[\frac{w_{0}^{2}}{\mu(z)} E_{0}(z, \theta) e^{i \theta_{j}}+\text { c.c. }\right]
\end{gathered}
$$

To obtain the governing equation for the Gaussian mode coefficient $E_{0}$, we insert the radiation decomposition (8) and the electron distribution function (7) in the paraxial equation (6) and use the orthogonality of the Gauss-Laguerre modes. Multiplying both sides by $e^{-\left(1+i z / z_{R}\right) x^{2} / 2 w^{2}}$ and integrating over the transverse dimension $\boldsymbol{x}$ yields

$$
\left[\frac{\partial}{\partial z}+k_{u} \frac{\partial}{\partial \theta}\right] E_{0}=-\frac{e K[J J]}{2 \pi \epsilon_{0} \gamma_{0}} \frac{N_{\lambda}}{\mu(z)^{*} \lambda}\left\langle e^{-i \theta_{j}}\right\rangle_{\lambda},
$$

where $\left\langle e^{-i \theta_{j}}\right\rangle_{\lambda}$ denotes the average of the ponderomotive phase over the $N_{\lambda}$ electrons in $\lambda$ :

$$
\left\langle e^{-i \theta_{j}}\right\rangle_{\lambda} \equiv \frac{1}{N_{\lambda}} \sum_{e_{j} \text { in } \lambda} e^{-i \theta_{j}} .
$$

The coupled FEL equations (12)-(14) include the lowestorder effects of electron beam emittance and radiation diffraction, and furthermore conserve the sum of the particle and field energies:

$$
\frac{d}{d z} \int d \theta\left(N_{\lambda}\left\langle\gamma_{0} m_{e} c^{2} \eta\right\rangle_{\lambda}+\pi \lambda w_{0}^{2} \epsilon_{0}\left|E_{0}\right|^{2}\right)=0 .
$$

The electron beam initially populates the phase $\theta$ randomly due to shot noise. To obtain the appropriate mean and variance of these initial fluctuations with a limited number of macroparticles, we weight the random departures in $\theta$ according to the noise algorithm of Penman and McNeil [13]. While the spontaneous radiation in the lowest-order transverse mode is reasonably well represented by our code (along with the subsequent longitudinal dynamics), neglecting other transverse modes leads to a significant underestimation of the total power in the spontaneous radiation (see, e.g., the discussion in [14]).

Finally, we note that, although the electron beam focusing parameter $z_{\beta}$ is independent of the radiation range $z_{R}$ (the former being set by the beam parameters while the latter is given by the cavity length and mirror curvature), the single-pass FEL gain is typically maximized when the radiation diffraction matches the beam spreading such that $z_{\beta} \approx z_{R} \approx N_{u} \lambda_{u} / 2 \pi[15]$.

\section{LINEAR SUPERMODE THEORY}

In this section we derive the growing modes of the x-ray FEL oscillator, i.e., the cavity supermodes. Supermode 
analysis of FELs was introduced in Ref. [9]; our work follows the simplified approach developed by Elleaume [10] as applied to the x-ray FEL oscillator in [3]. For the case of $\mathrm{x}$ rays of wavelength $\lambda$, the coherence time $\sigma_{c}$ of the undulator radiation is much less than the inverse bandwidth of the Bragg mirror $1 / \sigma_{\omega}$, so that slippage can be safely ignored; note that typically $\lambda / c \sigma_{c} \sim 1 / N_{u} \geq 10^{-3}$, while $\sigma_{\omega} \lambda / c \sim 10^{-5}-10^{-6}$. The radiation evolution during any pass then consists of FEL gain in the undulator followed by reflection from the narrow band Bragg mirrors, after which the process repeats on the next pass with a fresh electron bunch. At the beginning of the next pass the radiation pulse may be displaced in time with respect to the next electron bunch due to, for example, accelerating field timing jitter or a mismatched cavity length. We model this relative displacement by the cavity detuning length $c \ell$. We take the peak linear FEL gain to be $|g|<1$, the longitudinal electron beam profile to be a Gaussian with width $\sigma_{e}$, and the mirror reflection transfer function to be given by $R(\omega)$. In this case the evolution of the electric field amplitude $E$ over one pass can be depicted by the following three steps:

$$
\begin{gathered}
\text { gain: } E(\tau) \rightarrow\left[1+\frac{g}{2} e^{-\tau^{2} / 2 \sigma_{e}^{2}}\right] E(\tau), \\
\text { mirror: } E(\tau) \rightarrow \frac{1}{2 \pi} \int d \omega e^{-i \omega \tau} \tilde{R}(\omega) \tilde{E}(\omega),
\end{gathered}
$$

displacement: $E(\tau) \rightarrow E(\tau+\ell)$,

where, for the average beam velocity $\bar{v}$, the comoving bunch coordinate $\tau \equiv t-z / \bar{v}$, while $\tilde{E}(\omega)$ is the Fourier transform of $E(\tau)$. Note that we have removed the carrier frequency of the radiation from the electric field amplitude, so that $\omega$ is the frequency deviation from $\omega_{0}$, and the Fourier transform $\tilde{E}(\omega)$ is centered near zero.

To simplify the problem further, we make several approximations. First, we assume that the linear gain $g$ is small with $\mathfrak{R}(g)>0$ and $\Re(g) \gg|\Im(g)|$ (except where noted, we interpret our results using the approximation $\Im(g)=0)$, and expand the Gaussian beam profile about its maximum. Next, we model the narrow bandpass system of Bragg mirrors by a Gaussian filtering in frequency space with rms power bandpass given by $\sigma_{\omega}$. Assuming a small cavity loss of $\alpha$ due to absorption and transmission, the mirror transfer function $\tilde{R}(\omega) \approx(1-\alpha / 2) e^{-\omega^{2} / 4 \sigma_{\omega}^{2}}$, with $\omega$ representing the frequency difference from resonance. Typically, the x-ray Bragg mirrors have a bandpass $\sigma_{\omega}$ that is much larger than the transform-limited bandwidth of the electron beam $\sim 1 / \sigma_{e}$, in which case $\tilde{R}(\omega)$ is well described by its Taylor expansion to order $\omega^{2}$. In this approximation, the frequency filtering of the mirrors maps to a second-order derivative in the time domain. Finally, we assume that the detuning $\ell$ is sufficiently small to make a Taylor expansion here as well. Under these assumptions,
Eqs. (17)-(19) are simplified to

$$
\begin{aligned}
& \text { gain: } E(\tau) \rightarrow\left(1+\frac{g}{2}-\frac{g \tau^{2}}{4 \sigma_{e}^{2}}\right) E(\tau), \\
& \text { mirror: } E(\tau) \rightarrow\left(1-\frac{\alpha}{2}+\frac{1}{4 \sigma_{\omega}^{2}} \frac{\partial^{2}}{\partial \tau^{2}}\right) E(\tau),
\end{aligned}
$$

displacement: $E(\tau) \rightarrow\left(1+\ell \frac{\partial}{\partial \tau}\right) E(\tau)$.

We now use Eqs. (20)-(22) to relate the longitudinal radiation field starting pass number $n+1$ to that at the beginning of pass $n$. Assuming that the per-pass change is small, so that $E_{n+1}-E_{n} \approx \partial E / \partial n$, we find that the lowest-order evolution is described by the following partial differential equation:

$$
\begin{aligned}
\frac{\partial E}{\partial n}= & \frac{1}{4 \sigma_{\omega}^{2}} \frac{\partial^{2} E}{\partial \tau^{2}}+\ell \frac{\partial E}{\partial \tau}-\frac{\alpha}{2} E+\frac{g}{2}\left(1-\frac{\tau^{2}}{2 \sigma_{e}^{2}}\right) E \\
& +\mathcal{S}(\tau, n),
\end{aligned}
$$

where we have included the spontaneous undulator radiation of each pass with the stochastic source term $\mathcal{S}(\tau, n)$.

We determine the homogeneous solution of Eq. (23) by setting $\mathcal{S}=0$ and using the separation of variables technique. Defining $E(\tau, n)=\mathcal{A}_{1}(n) \mathcal{A}_{2}(\tau) e^{-2 \sigma_{\omega}^{2} \ell \tau}$, we separate the $n$ and $\tau$ dependencies, obtaining

$$
\begin{gathered}
\mathcal{A}_{1}(n)=\exp \left[\frac{1}{2}\left(g-\alpha-2 \sigma_{\omega}^{2} \ell^{2}\right)-\kappa\right], \\
\kappa \mathcal{A}_{2}(\tau)=-\frac{1}{4 \sigma_{w}^{2}} \frac{\partial^{2}}{\partial \tau^{2}} \mathcal{A}_{2}(\tau)+\frac{g}{4 \sigma_{e}^{2}} \tau^{2} \mathcal{A}_{2}(\tau),
\end{gathered}
$$

where $\kappa$ is the constant of separation. Thus, we see that $\mathcal{A}_{2}$ satisfies an eigenvalue equation whose solutions are the well-known Gauss-Hermite functions [note that the form of Eq. (25) is identical to that of the time-independent Schrödinger equation describing a simple harmonic oscillator]. The corresponding eigenvalue,

$$
\kappa=\left(m+\frac{1}{2}\right) \frac{\sqrt{g}}{2 \sigma_{e} \sigma_{\omega}},
$$

can be seen from (24) as reducing the effective per-pass gain. In the context of the FEL, this indicates that the gain approaches the infinite beam limit if the electron beam is significantly longer than the time given by the inverse bandwidth of the Bragg mirror $\left(\sigma_{e} \sigma_{\omega} \rightarrow \infty\right)$. For shorter electron bunches, only the fraction of the current whose spectral content lies within the mirror bandpass effectively contributes to the gain. Solving the eigenvalue equation (25) leads to a set $\left\{E_{m}\right\}$ of growing linear FEL modes, the so-called supermodes: 


$$
\begin{aligned}
E_{m}(\tau, n)= & e^{n \Lambda_{m} / 2} e^{-2 \sigma_{\omega}^{2} \ell \tau} \exp \left[-\frac{\sqrt{g} \sigma_{\omega}}{2 \sigma_{e}} \tau^{2}\right] \\
& \times H_{m}\left[g^{1 / 4} \sqrt{\frac{\sigma_{\omega}}{\sigma_{e}}} \tau\right]
\end{aligned}
$$

where $\Lambda_{m} \equiv\left[(g-\alpha)-2 \sigma_{\omega}^{2} \ell^{2}-\sqrt{g}(2 m+1) / 2 \sigma_{e} \sigma_{\omega}\right]$ is the per-pass power gain, and $H_{m}(x)$ is the Hermite polynomial of order $m$. From this, we deduce that in order to avoid significant gain reduction we must first demand the electron bunch-radiation timing errors to be much less than the inverse crystal bandwidth, typically $\ell \lesssim 0.1 / \sigma_{\omega}$. Additionally, higher-order modes have less gain, with $\Lambda_{m}<0$ for $\sigma_{e} \sigma_{\omega}<(m+1 / 2) \sqrt{g} /(g-\alpha)$; for the parameters of interest typically $2(g-\alpha) \lesssim g$, so we require $\sigma_{e} \gtrsim 1 /\left(\sigma_{\omega} \sqrt{g}\right)$ to ensure that at least the $m=0$ mode has sufficient per-pass gain for lasing.

We illustrate the dynamics of the FEL oscillator in Fig. 1, in which we plot the pass-to-pass supermode evolution as obtained from the 1D numerical FEL code described in Sec. II. The FEL parameters are those corresponding to the $12-\mathrm{keV}$ source listed in Table I, where the beam width $\sigma_{e}=1 \mathrm{ps}$, the cavity detuning $\ell$ is set to zero, and the electron focusing parameter $z_{\beta}$ and the Rayleigh range of the radiation $z_{R}$ are chosen to maximize the FEL gain: $z_{R}=z_{\beta}$. The power content in mode $m$ was extracted from the simulation by decomposing the real and imaginary parts of the longitudinal radiation profile into the Gauss-Hermite mode functions. The extracted exponential growth rates for the three lowest-order modes plotted in Fig. 1 differ by less than $10 \%$ from the theoretical power growth rate $\Lambda_{m}$ if we make the minor replacement $g \rightarrow g(1-\alpha)$; this correction ensures that the long pulse

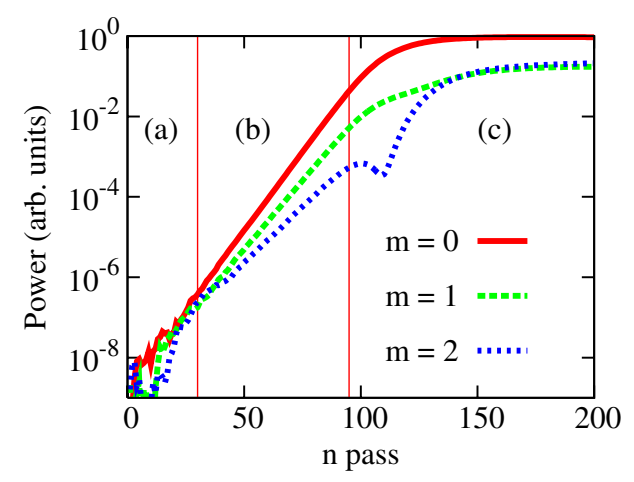

FIG. 1. (Color) Example of supermode growth for the lowest three order modes, using $12-\mathrm{keV}$ parameters of Table I with $\sigma_{e}=1 \mathrm{ps}$. The evolution of the modes can be roughly divided into three regions: region (a) gives the initial chaotic seeding from the spontaneous undulator radiation; region (b) depicts the linear growth of the supermodes, with the Gaussian $m=0$ mode having the largest growth; and region (c) is characterized by nonlinear saturation of the gain, where the growth of higherorder symmetric modes (e.g., $m=2$ ) reflects pulse broadening after saturation. limit growth rate $\Lambda_{m} \rightarrow[(1+g)(1-\alpha)-1]$ is recovered in the limit $\sigma_{e} \rightarrow \infty$.

The power content plotted in Fig. 1 clearly demonstrates the decrease in growth rate as the mode order $m$ increases. Additionally, we can identify three distinct regions of mode evolution, roughly delineated in Fig. 1 as regions (a), (b), and (c). Region (a) is dominated by the spontaneous undulator radiation that provides an initially chaotic seeding for the various modes. After the fluctuations have grown to a sufficient level, the exponential growth characteristic of linear gain can be seen in region (b). During this phase of evolution, the mode of order number $m$ exponentiates with the growth rate of $\Lambda_{m}$, with $\Lambda_{m}$ a decreasing function of $m$. Finally, when the radiation amplitude is sufficient to nonlinearly decrease the gain, we arrive at the saturation region (c). While the $m=0$ and $m=1$ modes level smoothly, we also see additional growth of the $m=2$ mode. Inspection of the longitudinal profile of the saturated radiation indicates that this corresponds to a broadening of the cavity pulse as the optical pulse width becomes closer to that of the electron beam $\sigma_{e}$.

The rest of this section further elaborates on the supermode properties. In the next subsection, we discuss how timing mismatches between the electron beam and the radiation (characterized by the detuning length $c \ell$ ) affects the growth and shape of the x-ray pulse. We then detail the physics of supermode seeding by the initially chaotic undulator radiation in the subsequent subsection. Finally, we conclude this section with some simulation results for the three sets of parameters listed in Table I obtained using our $1 \mathrm{D}$ code, and compare the $12-\mathrm{keV}$ evolution to that predicted by the two-dimensional FEL code GINGER.

\section{A. Timing errors and cavity detuning}

As indicated by (27), a displacement in time between the electron beam and the radiation gives rise to a skewing of the longitudinal $x$-ray profile along with a reduction in the single-pass linear gain. In simple physical terms, the mirror broadens the radiation in time, thereby increasing the temporal overlap with the next electron bunch and mitigating any reduction in gain. To be more precise, we note that the supermodes satisfy

$$
E_{m}(\tau, n)=e^{\Lambda_{m} / 2} E_{m}(\tau, n-1),
$$

namely, their longitudinal profile is invariant from pass-topass modulo the scale factor $e^{\Lambda_{m} / 2}$; indeed, this is how the supermodes were first defined in [9]. For the invariant mode profile $E_{m}$, we recall that the single-pass gain $\Lambda_{m}$ is maximized when its overlap between the electron beam and the Bragg mirror is maximized, namely, when it is centered with respect to the electron beam in $\tau$ and has an rms width proportional to the geometric mean of the electron beam width and the Bragg mirror correlation length [specifically, the supermode width equals $\sqrt{\sigma_{e} /\left(\sigma_{\omega} \sqrt{g}\right)}$ ]. 
While the detuning time $\ell$ tends to displace the radiation with respect to its previous pass, the narrow-bandwidth Bragg mirrors correlate the electric field over a time $\sim 1 / \sigma_{\omega}$. If this correlation time is much greater than the time lag induced by the cavity detuning, i.e., if $\sigma_{\omega} \ell \ll 1$, then the radiation profile is not significantly modified and the gain remains nearly unchanged. This is borne out by our supermode analysis, for which a displacement gives rise to a reduction in linear gain by $2 \sigma_{\omega}^{2} \ell^{2}$ and a displacement in $\tau$ of the supermode that scales as $\left(\sigma_{\omega} \ell\right) \sigma_{e}$.

We show how timing mismatches affect the 12-keV FEL oscillator performance in Fig. 2 (FEL parameters are listed in Table I). The first graph demonstrates the nearly quadratic decrease in gain with detuning $\ell$, where the crosses are obtained from the 1D simulation, and the line is given by supermode theory. The second graph plots the longitudinal pulse profile for four different values of the detuning $\ell$. Figure 2 indicates that performance is not significantly affected if timing errors are kept below $\sim 10$ fs (corresponding to a cavity detuning length of $\sim 1.5 \mu \mathrm{m}$ ).

Because the Bragg mirror bandwidth tends to increase as the photon energy decreases, these timing and cavity length tolerances tend to become more stringent at longer wavelengths. For the parameters listed in Table I, the timing between the electron beam and the radiation should be kept within $\sim 50$ fs $(\sim 12 \mu \mathrm{m})$ for the $20-\mathrm{keV}$ FEL, while $\ell<1$ fs $(\sim 0.15 \mu \mathrm{m})$ for the $5-\mathrm{keV}$ FEL (note how this also depends on the linear gain $g$ ). This rather stringent tolerance for the longer-wavelength FEL could be mitigated by increasing the linear gain $g$; for example, one might use additional bunch compression to increase the beam current.

In general, the FEL interaction leads to a complex amplitude gain, so that $g$ has real and imaginary components. In this case, the cavity detuning time $\ell$ additionally
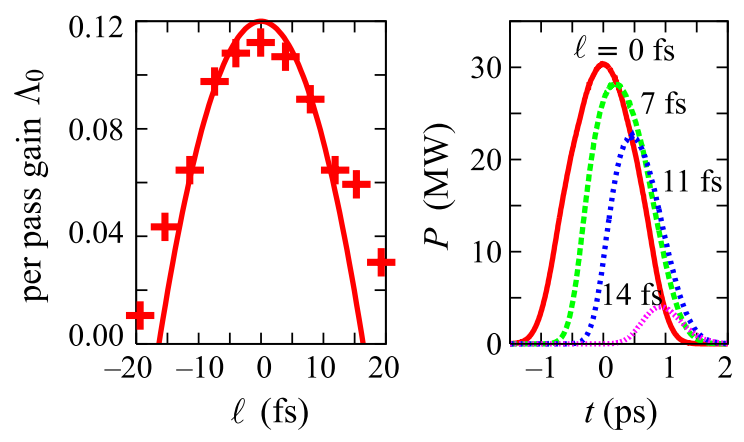

FIG. 2. (Color) Effect of timing mismatches between the electron beam and the radiation, i.e., detuning, for the $12-\mathrm{keV}$ example from Table I, with $\sigma_{e}=1 \mathrm{ps}$. The first graphs the single-pass gain measured from the 1D simulation (crosses) and from the supermode theory (lines). The second plots the longitudinal pulse profile after saturation for four different values of the detuning $\ell$. We see only modest pulse distortion and power reduction for $\ell \lesssim 10 \mathrm{fs}$, corresponding to a cavity length detuning of $1.7 \mu \mathrm{m}$. causes a shift in the central frequency of the amplified supermode. To calculate the magnitude of this effect, we consider here only the $m=0$ supermode. To determine the central frequency of the spectrum $\left|E_{0}(\omega)\right|^{2}$, we Fourier transform the $m=0$ mode of (27) and multiply by $\tilde{E}_{0}(\omega)^{*}$, assuming $g$ is now complex. After some simple manipulations, we find

$$
\left|\tilde{E}_{0}(\omega)\right|^{2} \propto \exp \left\{-\frac{\sigma_{e} \Re(\sqrt{g})}{\sigma_{\omega} g}\left[\omega-\frac{2 i\left(g-g^{*}\right) \sigma_{\omega}^{2} \ell}{g+2 g+g^{*}}\right]^{2}\right\},
$$

from which we see that the central frequency is unchanged if either $g$ is purely real or the detuning time $\ell=0$. For general gain and detuning, the $\mathrm{x}$-ray frequency is peaked near $\omega_{0}+\delta \omega$, with

$$
\delta \omega=\frac{2 i\left(g-g^{*}\right)}{g+2 g+g^{*}} \sigma_{\omega}^{2} \ell .
$$

To give an idea of the magnitude of this effect, we note that for the $12-\mathrm{keV}$ parameters of Table I we have $\Im(g) \sim$ $\Re(g) / 4$, so that for a diamond crystal near $1 \AA$ we have, in practical units

$$
|\delta \omega|[\mathrm{meV}] \sim 0.1 \times(c \ell)[\mu \mathrm{m}] .
$$

Finally, we comment that after saturation, $\delta \omega$ may be larger than that implied by (29) or (30) because the curvature near peak reflectivity of real Bragg crystals is larger than the rms width $\sigma_{\omega}$.

\section{B. Initial seeding from noise}

In this section we investigate the seeding of the linear supermodes by the spontaneous undulator radiation $\mathcal{S}(n, \tau)$, and calculate the statistical distribution of the modes during the initial phase of evolution [i.e., in region (a) of Fig. 1]. Previously, the FEL oscillator start-up from noise has been analyzed in some detail by $[16,17]$, while less complicated analytic expressions for the initial value problem were derived in Ref. [18] for an ideal (no energy spread, constant in time) electron beam in a Fabry-Perottype resonator. We describe the pass-to-pass evolution using the simplified model (23), so that our analysis more closely resembles that of Ref. [19]. However, we make explicit use of the supermode (Gauss-Hermite) eigenfunctions, and expand the electric field as

$$
E(\tau, n)=\sum_{q=0}^{\infty} \mathcal{E}_{q}(n) \frac{H_{q}(\tau / T)}{\sqrt{2^{q} q ! \sqrt{\pi}}} e^{\tau^{2} / 2 T^{2}},
$$

where $T^{2} \sqrt{g} \equiv \sigma_{e} / \sigma_{\omega}$ defines the supermode width. Inserting (31) into the differential equation (23), multiplying by the orthogonal Gauss-Hermite mode $e^{-\tau^{2} / 2 T^{2}} H_{m}(\tau / T)$ and integrating over $\tau$ yields the following Langevin equation for the mode coefficient $\mathcal{E}_{m}$ : 


$$
\begin{aligned}
\frac{\partial \mathcal{E}_{m}}{\partial n} & =\frac{\Lambda_{m}}{2} \mathcal{E}_{m}+\int d \tau \mathcal{S}(\tau, n) \frac{H_{m}(\tau / T)}{\sqrt{2^{m} m ! \sqrt{\pi}}} e^{-\tau^{2} / 2 T^{2}} \\
& \equiv \frac{\Lambda_{m}}{2} \mathcal{E}_{m}+\mathcal{S}_{m}(n)
\end{aligned}
$$

with $\mathcal{S}_{m}(n)$ a stochastic function of the pass number representing the spontaneous radiation that is reflected and filtered by the Bragg mirrors. Solution of (32) for $\mathcal{E}_{m}(0)=$ 0 is straightforward:

$$
\mathcal{E}_{m}(n)=e^{n \Lambda_{m} / 2} \int_{0}^{n} d t \mathcal{S}_{m}(t) e^{-\Lambda_{m} t / 2} .
$$

Thus, the power in each mode initially grows according to its overlap with the spontaneous radiation given by $\mathcal{S}_{m}$. To obtain the ensemble averaged power from Eq. (33), we assume that the spontaneous radiation is statistically independent of the pass number and uncorrelated from pass to pass (i.e., stationary and $\delta$-correlated in $n$ ). For any function $f$, this implies that

$$
\begin{aligned}
\int d t f(t)\left\langle\mathcal{S}_{m}(n) \mathcal{S}_{m}^{*}(n+t)\right\rangle & =\int d t f(t)\left\langle\mathcal{S}_{m}(0) \mathcal{S}_{m}^{*}(t)\right\rangle \\
& =f(0)\left\langle\mathcal{S}_{m}(0) \mathcal{S}_{m}^{*}(0)\right\rangle,
\end{aligned}
$$

where $\langle\cdot\rangle$ denotes the ensemble average over many instances of the oscillator start-up from noise. Using the statistics (34), the pass evolution of the ensemble averaged power contained in each mode follows naturally from (33), and is given by

$$
\left\langle\left|\mathcal{E}_{m}(n)\right|^{2}\right\rangle=\left\langle\left|\mathcal{S}_{m}(0)\right|^{2}\right\rangle\left(e^{n \Lambda_{m}}-1\right) / \Lambda_{m} .
$$

Thus, the ensemble averaged power in mode $m$ grows exponentially with growth rate $\Lambda_{m}$ and initial seeding power given by the average overlap of the supermode with the reflected spontaneous radiation. Furthermore, (35) and (33) indicate that the exponential gain becomes the principal contribution after $n \sim 1 / \Lambda_{m}$ passes; for typical values the exponential stage [region (b) of Fig. 1] begins after $1 / \Lambda_{0} \sim 5-50$ passes. During the initial phase [Fig. 1, region (a)], on the other hand, the uncorrelated source term dominates and the complex supermode coefficients undergo a random walk in amplitude with a "step size" proportional to its overlap with $\mathcal{S}(\tau)$. Thus, in the initial seeding stage the power in each mode $\propto\left|\mathcal{E}_{m}\right|^{2}$ tends to increase linearly with pass number, with the growth for each mode $m$ proportional to the undulator radiation power contained in that mode, as indicated by (35) for $n<1 / \Lambda_{m}$. Furthermore, the incoherent power in each mode will fluctuate significantly about this average linear increase depending on the precise phasing of each single-pass contribution. The power in each supermode increases in this noisy, nearly linear fashion until the power in the coherently amplified component is larger than the spontaneous signal $|\mathcal{S}|^{2}$ (or, in the case that $\Lambda_{m}<0$, until the spontaneous power in the mode balances the single-pass loss).
As discussed above, the power in each mode initially grows according to its overlap with the Bragg-reflected spontaneous radiation $\mathcal{S}(\tau, n)$. Thus, to determine the FEL seeding power of the $m$ th mode we must calculate what fraction of the single-pass power $|\mathcal{S}|^{2}$ is contained in the mode $m$. We model the envelope of the chaotic spontaneous radiation as a random collection of Gaussian spikes, each of whose width is the undulator coherence time $\sigma_{c} \sim$ $N_{u} \lambda / c$ :

$$
\begin{aligned}
& \mathcal{S}(\tau, n)=\sum_{j} \mathcal{E}_{0} \exp \left[-\frac{\left(\tau-\tau_{j}\right)^{2}}{4 \sigma_{c}^{2}}+i \omega_{0} \tau_{j}\right] \\
& \Rightarrow \tilde{\mathcal{S}}(\omega, n)=\sqrt{4 \pi} \sigma_{c} \mathcal{E}_{0} \sum_{j} e^{-\sigma_{c}^{2} \omega^{2}+i\left(\omega+\omega_{0}\right) \tau_{j}} .
\end{aligned}
$$

In Eqs. (36) and (37), $j$ indexes the bunch electrons, the phase difference $\omega_{0} \tau_{j}$ accounts for the individual entrance times of the electrons in the undulator, and $\mathcal{E}_{0}$ can be obtained from the well-known theory of undulator radiation. Upon reflection in the cavity, the Bragg crystal effectively filters the radiation in frequency space as given by (18), where we approximate $\tilde{R}(\omega)$ as a lossy Gaussian filter with width $\sigma_{\omega}$ and power loss $\alpha$. Thus, after reflection and filtering by the two Bragg mirrors, the Fourier transform of the spontaneous radiation (37) becomes

$$
\begin{aligned}
\tilde{\mathcal{S}} & \rightarrow \sqrt{1-\alpha} e^{-\omega^{2} / 4 \sigma_{\omega}^{2}} \tilde{\mathcal{S}}(\omega, n) \\
& \approx \sqrt{4 \pi} \sigma_{c}\left(1-\frac{\alpha}{2}\right) \mathcal{E}_{0} \sum_{j} e^{-\omega^{2} / 4 \sigma_{\omega}^{2}+i\left(\omega+\omega_{0}\right) \tau_{j},}
\end{aligned}
$$

where the second line follows by assuming that the mirror bandwidth is much less than that of the undulator radiation $\left(\sigma_{\omega}^{2} \ll 1 / \sigma_{c}^{2}\right)$, and that the loss $\alpha$ is small. The overlap of the initially chaotic spontaneous undulator radiation with the supermode eigenfunctions is defined by (32). Because the Gauss-Hermite basis is diagonal with respect to the Fourier transform operator, this integral can be expressed as

$$
\mathcal{S}_{m}=\int d \omega \frac{\tilde{\mathcal{S}}(\omega, n)}{i^{m} \sqrt{2 \pi}} \frac{H_{m}(\omega T)}{\sqrt{2^{m} m ! \sqrt{\pi}}} e^{-\omega^{2} T^{2} / 2} .
$$

Inserting the expression (38) for $\tilde{\mathcal{S}}(\omega, n)$ and taking the integral with respect to $\omega$ (see, e.g., Gradshteyn and Ryzhik [20]), the mode coefficients are given by

$$
\begin{aligned}
\mathcal{S}_{m}= & \mathcal{E}_{0} \frac{2 \sigma_{c} \sqrt{\pi}\left(1-\frac{\alpha}{2}\right)}{\sigma_{+} \sqrt{2^{m} m ! \sqrt{\pi}}} \frac{\sigma_{-}^{m}}{\sigma_{+}^{m}} \times \sum_{j} \exp \left[-\frac{\tau_{j}^{2}}{2 \sigma_{+}^{2}}+i \omega_{0} \tau_{j}\right] \\
& \times H_{m}\left(\frac{T \tau_{j}}{\sigma_{+} \sigma_{-}}\right),
\end{aligned}
$$

where, for compactness, we have defined the widths

$$
\sigma_{ \pm}^{2} \equiv T^{2} \pm \frac{1}{2 \sigma_{\omega}^{2}} \equiv \frac{\sigma_{e}}{\sqrt{g} \sigma_{\omega}} \pm \frac{1}{2 \sigma_{\omega}^{2}} .
$$


We note $\sigma_{e} \sigma_{\omega}>1 / \sqrt{g}>\sqrt{g}$ to have FEL gain, so that $\sigma_{ \pm}^{2}>0$ for all parameters of interest.

The coefficients $\mathcal{S}_{m}$ in (40) give the overlap between the initial filtered undulator radiation and the supermodes of order $m$. The initially stochastic radiation seeds each mode with a power level proportional to $\left|\mathcal{S}_{m}\right|^{2}$ given by

$$
\begin{aligned}
\left|\mathcal{S}_{m}\right|^{2}= & \frac{(1-\alpha)\left|\mathcal{E}_{0}\right|^{2} 4 \pi \sigma_{c}^{2}}{2^{m} m ! \sqrt{\pi} \sigma_{+}^{2}} \frac{\sigma_{-}^{2 m}}{\sigma_{+}^{2 m}} \\
& \times\left\{\sum_{j} e^{-\tau_{j}^{2} / \sigma_{+}^{2}} H_{m}^{2}\left(\frac{T \tau_{j}}{\sigma_{+} \sigma_{-}}\right)\right. \\
& \left.+\sum_{j \neq k} e^{i \omega_{0}\left(\tau_{j}-\tau_{k}\right)}[\cdots]\right\} .
\end{aligned}
$$

The second sum in (41) is significant if there is electron structure (bunching) at scale lengths on the order of the radiation wavelength. For undulator radiation, however, the electron bunching is small, and the contributions from the rapidly varying phase $\omega_{0}\left(\tau_{j}-\tau_{k}\right)$ largely cancel in comparison with the first sum. We calculate the spontaneous power by replacing the sum with an integral over the particle distribution via

$$
\sum_{j} \rightarrow N_{e} \int d \tau_{j} \frac{e^{-\tau_{j} / 2 \sigma_{e}^{2}}}{\sqrt{2 \pi} \sigma_{e}}
$$

and neglect the second contribution, so that the spontaneous power in the mode $m$ is given by

$$
\begin{aligned}
\left|\mathcal{S}_{m}\right|^{2}= & (1-\alpha) N_{e}\left|\mathcal{E}_{0}\right|^{2} \frac{2 \sqrt{2} \sigma_{c}^{2}}{2^{m} m ! \sigma_{e} T} \frac{\sigma_{-}^{2 m+1}}{\sigma_{+}^{2 m+1}} \\
& \times \int d x \exp \left[-\frac{\left(2 \sigma_{e}^{2}+\sigma_{+}^{2}\right) \sigma_{-}^{2}}{2 \sigma_{e}^{2} T^{2}} x^{2}\right] H_{m}^{2}(x) .
\end{aligned}
$$

The integral in (42) can be evaluated analytically in terms of Gauss's hypergeometric function. We reserve this calculation for the Appendix, finding it instructive to approximate the integral in the following manner. As discussed previously, FEL gain requires $\sigma_{e}>T>1 / \sigma_{\omega}$, and we typically have $\sigma_{e}^{2} \gg \sigma_{+}^{2} \approx T^{2} \approx \sigma_{-}^{2}>1 / \sigma_{\omega}^{2}$. In this limit the argument of the exponential in (42) becomes $-x^{2}$ and the integral equals $2^{m} m$ !, so that the power in mode $m$ scales as $\left(\sigma_{-} / \sigma_{+}\right)^{2 m+1}$. This implies that the filtered undulator radiation provides significant seeding for modes of order $m \lesssim \sigma_{e} \sigma_{\omega} / \sqrt{g}$. Note that while this condition scales in the same manner with respect to the electron beam and mirror width as does the condition for mode $m$ to have positive FEL gain, the latter requirement $m \lesssim(g-\alpha) \sigma_{e} \sigma_{\omega} / \sqrt{g}$ is more stringent.

To streamline our presentation, we leave the evaluation of (42) for arbitrary $m$ to the Appendix and instead explicitly consider the relative initial seeding of the two lowestorder averaged-mode coefficients $\left|\mathcal{S}_{0}\right|^{2}$ and $\left|\mathcal{S}_{1}\right|^{2}$. These are of particular interest because they have the largest linear gain and typically have the largest initial seeding. By evaluating (42) [or alternatively (A1) in the Appendix] for $m=0$ and $m=1$ we find

$$
\begin{aligned}
\frac{\left|\mathcal{S}_{0}\right|^{2}}{\left|\mathcal{S}_{1}\right|^{2}} & =\frac{4 \sigma_{-}^{2} \sigma_{e}^{2}+\sigma_{+}^{2} \sigma_{-}^{2}-\sigma_{e}^{2} / \sigma_{\omega}^{2}}{4 \sigma_{e}^{2} \sigma_{+}^{2}+2 \sigma_{+}^{2} \sigma_{+}^{2}} \\
& \approx 1-\frac{1+g}{4 \sqrt{g} \sigma_{e} \sigma_{\omega}}+O\left(1 / \sigma_{e}^{2} \sigma_{\omega}^{2}\right),
\end{aligned}
$$

indicating that the spontaneous radiation from a single pass tends to seed the two lowest-order supermodes about equally. On the next pass, the spontaneous radiation $\mathcal{S}$ will have a similar expansion in terms of the mode coefficients but will differ by an uncorrelated change in the overall phase; in the model (36), this would correspond to $\mathcal{E}_{0}$ having a random phase from pass to pass. As mentioned earlier, after $n$ passes in the initial stage dominated by the spontaneous contribution (i.e., for $n \lesssim 1 / \Lambda_{0}$ before linear gain takes over), we have

$$
\left\langle\left|\mathcal{S}_{0}(n)\right|^{2}\right\rangle \approx\left\langle\left|\mathcal{S}_{1}(n)\right|^{2}\right\rangle \approx n\left\langle\left|\mathcal{S}_{0}\right|^{2}\right\rangle
$$

Although the ensemble-averged spontaneous power increases linearly with pass number, the fluctuations in mode power between different instances of the oscillator start-up are close to $100 \%$ before the signal is dominated by gain. Therefore, while on average the lowest-order modes are equally seeded by the undulator radiation $\mathcal{S}$, on occasion the $m=1$ is preferentially seeded so that the pulse is no longer largely Gaussian in the linear gain regime [i.e., region (b) of Fig. 1]. We show an example of this phenomenon in Fig. 3. The first graph demonstrates that the initial fluctuating phase [region (a)] can sometimes dominantly seed the first-order mode. The linear growth phase is then initially dominated by the antisymmetric $m=1$ mode through region (b1). As the amplification continues, however, the radiation approaches a Gaussian due to the higher growth rate of the $m=0$ mode, which then becomes the dominant component throughout region (b2).

We also show two examples of complex electric field amplitudes in the linear gain region in Fig. 3. After 40 passes we see that both the real (red solid lines) and imaginary (blue dotted lines) parts of $E$ are largely described by the $m=1$ supermode; after 80 passes the pulse has more overlap with the $m=0$ mode, although there is still a significant antisymmetric component from the mode with $m=1$. As evolution continues, the pulse becomes more symmetric, until it is nearly Gaussian at saturation.

\section{Examples for a 7-GeV beam}

In this section we present supermode evolution results relevant to a low-emittance, $7-\mathrm{GeV}$ electron beam whose current is $10 \mathrm{~A}$ and whose electron beam and undulator parameters are listed in Table I. For this study we have 

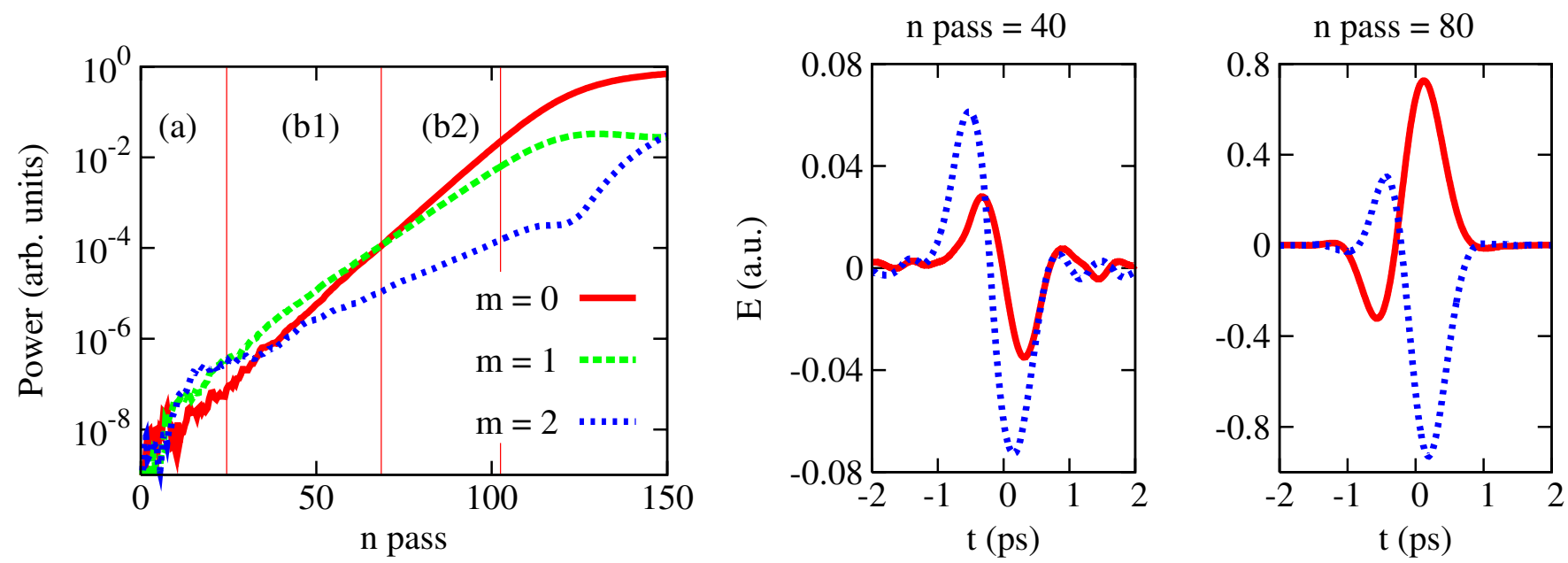

FIG. 3. (Color) Example where the initial random seeding is largest in the $m=1$ mode, using the same parameters (but different seeding) as in Fig. 1. The first plot shows initial seeding of the first-order mode in region (a), after which the linear growth phase is dominated by the $m=1$ mode through region (b1). Because of its larger growth rate, the component of the $m=0$ mode eventually becomes dominant in region (b2). We show examples of the real (red solid line) and imaginary (blue dotted line) parts of the scaled electric field after 40 passes and after 80 passes. While there is still a significant antisymmetric part after 80 passes, it becomes a diminishing component, and at saturation the pulse is nearly Gaussian.

taken Bragg mirror bandwidths that approximate those of perfect diamond mirrors, for which the peak reflectivity can be made $\sim 99 \%$. We assume a total per-pass cavity loss $\alpha=0.15$; this could arise, for example, from a fewpercent energy absorption of the perfect crystal mirrors, a $4 \%$ decrease due to outcoupling of the radiation from the cavity, and an additional 5\% loss per pass for each of two focusing elements.

Additionally, we include in Table I the saturated power $P_{\text {sat }}$ for a 1-ps electron beam as predicted by the 1D model introduced in Sec. II, and compare that to the saturated power predicted by the 2D code GINGER. We find that our 1D code agreed with GINGER in terms of the saturated power to within $10 \%-30 \%$, with the outcoupled radiation predicted to be of order 0.5-10 MW. Furthermore, we observe that after $\sim 100$ passes the pulse broadens in time due to the nonlinear saturation mechanism, while the bandwidth decreases such that the rms time-frequency product $\Delta \tau \Delta \omega$ is nearly constant. After many passes we find that the radiation temporal duration is of order $\sim \sigma_{e} / 2$, the radiation bandwidth is much narrower than the mirror bandwidth $\sigma_{\omega}$, and the pulse is nearly Fourier limited: $\Delta \tau \Delta \omega \approx 1.5$.

We summarize the supermode evolution for all three photon energies in Fig. 4, in which we plot the measured and theoretical per-pass gain for the lowest two order modes as a function of the electron bunch length $\sigma_{e}$. To determine the mode growth rates, after each pass we decompose the real and imaginary parts of $E(\tau)$ into the Gauss-Hermite basis given by (27). Plotting the absolute square of these mode coefficients as a function of pass number yields plots similar to Figs. 1 and 3, from which the growth rate can be determined by a least squares fit.
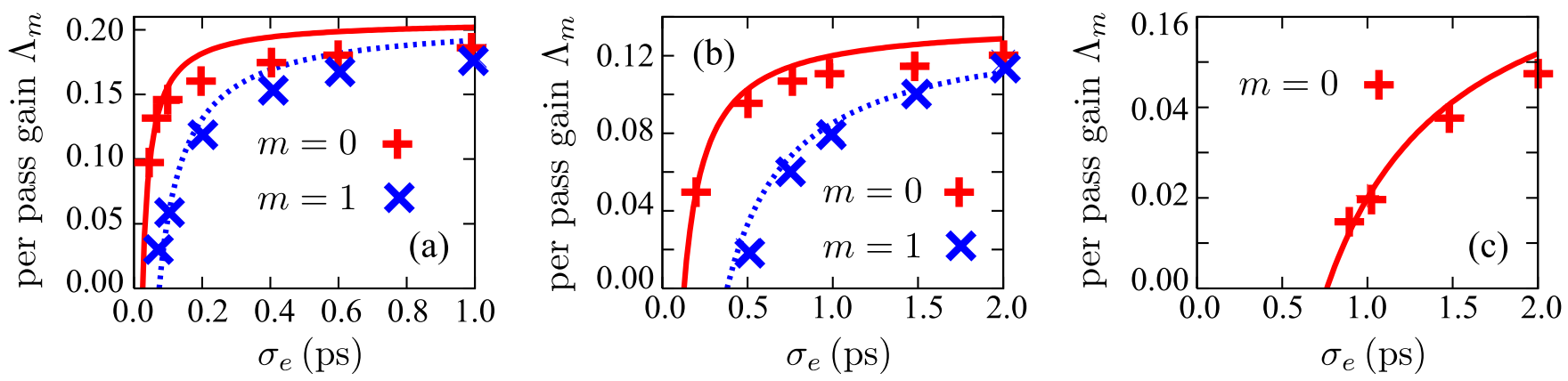

FIG. 4. (Color) Supermode gain rates for the 5-keV (a), 12-keV (b), and 20-keV (c) radiation cases detailed in Table I, using a per-pass cavity loss $\alpha=0.15$. Simulation points were determined by decomposing the longitudinal radiation onto the Gauss-Hermite modes and are plotted for the lowest two order modes when they could be determined (and are nonzero). Theory is given by the lines, where the power growth rate $\Lambda_{m} \equiv\left[(g-\alpha)-\sigma_{\omega}^{2} \ell^{2} / 2-\sqrt{g}(2 m+1) / \sigma_{e} \sigma_{\omega}\right]$, and we have replaced $g \rightarrow(1-\alpha) g$ so that $\Lambda_{m}$ reduces to the infinite pulse per-pass gain of $[(1+g)(1-\alpha)-1]$. 
Figure 4 contains measured growth rates for the Gaussian $(m=0)$ mode and the lowest-order antisymmetric $(m=1)$ mode for the 5-keV (a), 12-keV (b), and 20$\mathrm{keV}$ (c) FEL designs. In each scenario the per-pass gain decreases as the bunch length decreases, until reaching zero gain when $\sigma_{e} \sim 1 / \sigma_{\omega} \sqrt{g}$. Since the gain decreases more rapidly for the higher-order modes, the cavity pulse becomes more Gaussian as the electron bunch length decreases. Furthermore, because the crystal bandwidth decreases as the photon energy increases, this gain reduction is more significant at the higher photon energies, so much so that only the $m=0$ mode has positive growth for the 20-keV FEL in panel (c).

Finally, we indicate how the one-dimensional supermode theory presented here continues to be of use when one considers pulses with self-consistently evolving transverse dynamics. In general, such an analysis involves the full set of transverse modes supported by the cavity which for $g<1$ can be approximated by the vacuum GaussLaguerre optical modes (see, e.g., Siegman [21] for a full discussion). Because the single-pass gain $g$ is maximized when the radiation profile matches that of the electron beam (see the gain formula in [15] and discussion in [22]), the FEL preferentially selects the lowest-order, Gaussian transverse mode. By projecting the transverse radiation profile at each longitudinal position onto this cavity mode, one can effectively reduce the radiation to a 1D field suited for our supermode analysis.

We have carried out this transverse projection prescription to study the longitudinal supermode evolution with the 2D simulation code GINGER. After the initial stage, which is dominated by the chaotic undulator radiation $(\sim 5-20$ passes), we have found that nearly $99 \%$ of the radiation power is contained in the lowest-order transverse mode of the cavity. Having projected the field onto what is now one longitudinal dimension, we can perform the same super-

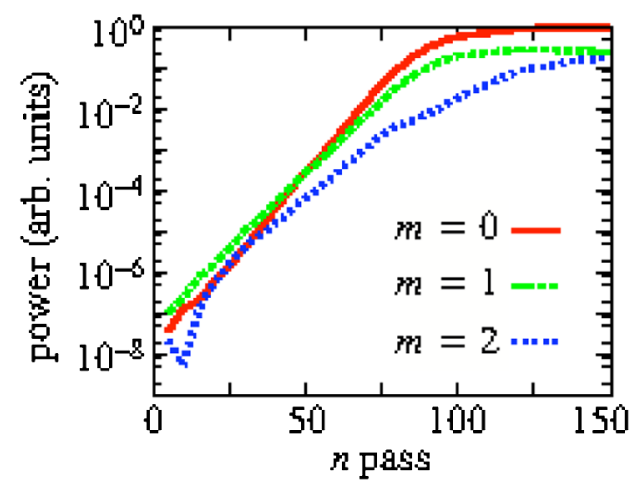

FIG. 5. (Color) Example of supermode growth for the lowest three order modes obtained from a 2D GINGER simulation of the $12-\mathrm{keV}$ FEL in Table I. The transverse field is projected onto the Gaussian cavity mode, thereby allowing a 1D supermode analysis. Note how the $m=1$ mode initial dominates, but the radiation evolves to be nearly Gaussian because of better coupling to the $m=0$ mode. mode decomposition. An example of the resulting supermode growth is plotted in Fig. 5, for the same parameters as Fig. 1, namely, the 12-keV FEL detailed in Table I with $\sigma_{e}=1 \mathrm{ps}$. We see that the two figures look quite similar, with both indicating a decrease in growth rate as the mode order $m$ increases, and significant content in the $m=2$ mode after saturation indicating pulse broadening. The main difference is in the initial chaotic stage: the 1D code indicates large mode fluctuations due to the chaotic undulator radiation for $\sim 20$ passes, while the 2D GINGER simulations seem to exit this initial stage much earlier, after only 5 passes or so. Nevertheless, the subsequent dynamics are quite similar and can even show evidence of dominantly seeding the $m=1$ mode, as previously discussed. Furthermore, the general conclusions regarding the dependence of the single-pass gain on both the electron beam length and on the timing between the radiation and the subsequent electron bunch hold.

\section{CONCLUSIONS}

We have shown that the linear supermodes of the X-ray FEL oscillator are the growing Gauss-Hermite modes whose per-pass growth rate decreases for increasing mode order and for decreasing electron bunch width. Seeding of these modes by the chaotic undulator radiation seeds is random, so that the initial evolution may be dominated by higher-order longitudinal modes. Nevertheless, the lowest-order $m=0$ Gaussian mode eventually dominates due to its larger growth rate, so that the final longitudinal radiation profile is nearly Gaussian at saturation.

\section{ACKNOWLEDGMENTS}

The authors wish to thank W. M. Fawley for many useful discussions on FEL simulations, and Y. Shvyd'ko for his expertise with $\mathrm{x}$-ray optics. This work was supported by U.S. Department of Energy, Office of Science, Office of Basic Energy Sciences, Contract No. DE-AC0206CH11357.

\section{APPENDIX: INITIAL SEEDING OF ARBITRARY SUPERMODES}

In this Appendix we explicitly evaluate and simplify the integral expression (42) for arbitrary $m$. The integral can be found in standard tables [20] expressed as

$$
\begin{aligned}
& \int d x e^{-\alpha^{2} x^{2}} H_{m}^{2}(x) \\
& =\frac{4^{m}}{\alpha \sqrt{2}}\left(\frac{1-\alpha^{2}}{\alpha^{2}}\right)^{m} \\
& \quad \times \Gamma\left(m+\frac{1}{2}\right)_{2} F_{1}\left(-m,-m ; \frac{1}{2}-m ; \frac{\alpha^{2} / 2}{\alpha^{2}-1}\right) .
\end{aligned}
$$

To make this formula practical, we use the hypergeometric 
function series expansion

$$
\begin{aligned}
{ }_{2} F_{1}\left(-m,-m ; \frac{1}{2}-m ; \xi\right) \equiv & \sum_{k=0}^{\infty} \frac{(-m)_{k}(-m)_{k}}{\left(\frac{1}{2}-m\right)_{k} k !} \xi^{k} \\
= & \sum_{k=0}^{m} \frac{(m !)^{2}}{[(m-k) !]^{2} m} \\
& \times \frac{\Gamma\left(\frac{1}{2}-m\right)}{\Gamma\left(\frac{1}{2}-m+k\right)} \xi^{k}
\end{aligned}
$$

The second line above arises from the definition of the Pochhammer symbol $(a)_{k}$ and its subsequent reduction when $a$ is a negative integer. Additionally, we use the gamma function identity

$$
\Gamma\left(\frac{1}{2} \pm n\right)=( \pm 1)^{n} \sqrt{\pi}\left[\frac{(2 n-1) ! !}{2^{m}}\right]^{ \pm 1}
$$

where we define $(-1) ! ! \equiv 1$. Further simplification obtains by replacing the summation variable $k$ with $m-k$; collecting the various pieces, we find that the power of the spontaneous radiation $\mathcal{S}(\tau)$ contained in the $m$ th mode is proportional to

$$
\begin{aligned}
\left|\mathcal{S}_{m}\right|^{2}= & (1-\alpha) N_{e}\left|\mathcal{E}_{0}\right|^{2} \frac{4 \sqrt{\pi} \sigma_{c}^{2}}{\sigma_{+}\left(2 \sigma_{e}^{2}+\sigma_{+}^{2}\right)^{1 / 2}} \frac{\sigma_{-}^{2 m}}{\sigma_{+}^{2 m}} \\
& \times \sum_{k=0}^{m} \frac{m !(2 k-1) ! !}{(k !)^{2}(m-k) !}\left[-\frac{\sigma_{+}^{2} \sigma_{-}^{2}-\sigma_{e}^{2} / \sigma_{\omega}^{2}}{2 \sigma_{-}^{2}\left(2 \sigma_{e}^{2}+\sigma_{+}^{2}\right)}\right]^{k} .
\end{aligned}
$$

The seeding of the mode coefficients decreases with $m$ as the supermode width increases, becoming very small when the $m$ th supermode width approaches (from below) that of the electron beam, i.e., when $m \sim \sqrt{g} \sigma_{e} \sigma_{\omega}$.

[1] C. Brau, Free-Electron Lasers (Academic Press, New York, 1990).
[2] R. Colella and A. Luccio, Opt. Commun. 50, 41 (1984).

[3] K.-J. Kim, Y. Shvyd'ko, and S. Reiche, Phys. Rev. Lett. 100, 244802 (2008).

[4] K.-J. Kim and Y. Shvyd'ko, Phys. Rev. ST Accel. Beams 12, 030703 (2009).

[5] Y. Shvyd'ko, X-Ray Optics-High Energy-Resolution Applications (Springer, New York, 2004).

[6] LCLS conceptual design report. Stanford Linear Accelerator Center, Technical Report No. SLAC-R-593, 2002.

[7] K. Halbach, J. Phys. (Paris) 44, C1 (1983).

[8] W. M. Fawley, Lawrence Berkeley Laboratory Technical Report No. LBNL-49625, 2002.

[9] G. Dattoli, A. Marino, A. Renieri, and F. Romanelli, IEEE J. Quantum Electron. 17, 1371 (1981).

[10] P. Elleaume, IEEE J. Quantum Electron. 21, 1012 (1985).

[11] S. Reiche, Nucl. Instrum. Methods Phys. Res., Sect. A 429, 243 (1999).

[12] Z. Huang and K.-J. Kim, Phys. Rev. ST Accel. Beams 10, 034801 (2007).

[13] C. Penman and B. W. J. McNeil, Opt. Commun. 90, 82 (1992).

[14] W. M. Fawley, Nucl. Instrum. Methods Phys. Res., Sect. A 507, 19 (2003).

[15] K.-J. Kim, Nucl. Instrum. Methods Phys. Res., Sect. A 318, 489 (1992).

[16] P. Sprangle, C. M. Tang, and I. Bernstein, Phys. Rev. A 28, 2300 (1983).

[17] G. Shvets and J. S. Wurtele, Nucl. Instrum. Methods Phys. Res., Sect. A 393, 273 (1997).

[18] The Physics of Free-Electron Lasers, edited by E. L. Saldin, E. A. Schneidmiller, and M. V. Yurkov (SpringerVerlag, Berlin, 2000).

[19] K.-J. Kim, Phys. Rev. Lett. 66, 2746 (1991).

[20] Table of Integrals, Series, and Products, edited by I. S. Gradshteyn and I. M. Ryzhik (Academic Press, New York, 1980).

[21] A. E. Siegman, Lasers (University Science Books, New York, 1986).

[22] K.-J. Kim, Nucl. Instrum. Methods Phys. Res., Sect. A 246, 71 (1986). 